\title{
Investigación sobre psicoterapia en abuso sexual infantil: ¿Una tarea pendiente en Chile?
}

\author{
Psychotherapy research with sexually abused children: A pending issue \\ in Chile?*
}

\author{
Cristobal Guerra** \\ Pontificia Universidad Católica de Chile. \\ Valeria Arredondo \\ Centro de Estudios en Infancia, Adolescencia y Familia de la ONG Paicabí, \\ Viña del Mar, Chile. \\ (Rec.: enero de 2015 - Acept.: julio de 2016)
}

\begin{abstract}
Resumen
El abuso sexual infantil es un fenómeno de alta prevalencia que genera consecuencias negativas en el desarrollo de los niños expuestos a sus diversas formas. El objetivo de este trabajo es analizar la producción nacional de estudios referidos a la psicoterapia con víctimas de abuso sexual infantil. La muestra la componen ocho revistas y seis instituciones chilenas que trabajan con víctimas. De los solo 19 trabajos en el área, la mayoría se corresponden a la sistematización de experiencias de intervención. Igualmente, se aprecia una escasez de estudios empíricos. Con lo cual se manifiesta el desafío a las instituciones e investigadores de desarrollar estudios con el rigor metodológico necesario para generar conocimiento en el contexto nacional que nutra la labor de los psicoterapeutas que trabajan con víctimas.
\end{abstract}

Palabras clave: investigación, psicoterapia, abuso sexual, infancia.

\begin{abstract}
Child sexual abuse is an outweighing phenomenon that generates negative consequences on children's development. The aim of this research is to analyse the national research on psychotherapy with victims of child sexual abuse. Eight journals and six Chilean institutions form the research sample. From the only 19 studies in the area, the majority focus on the systematisation of experiences of intervention. Furthermore, there are few empiric studies. This highlights the challenge for institutions and researchers to conduct research with the required methodological rigour to enhance knowledge in the national context to provide assistance to psychotherapists working with these victims.
\end{abstract}

Keywords: research, psychotherapy, sexual abuse, childhood.

\footnotetext{
* Este artículo fue realizado con los aportes del Programa de Formación de Capital Humano Avanzado de Conicyt del Gobierno de Chile, por medio de la Beca doctoral otorgada al primer autor.

** Correspondencia a: Cristobal Guerra. Pontificia Universidad Católica de Chile, Santiago. E-mail: cguerravio@uc.cl.
} 


\section{Introducción}

El abuso sexual infantil (ASI) corresponde a cualquier contacto sexual mantenido entre un adulto y un menor de edad o bien entre menores de edad cuando existe asimetría de poder entre ambos o algún tipo de coacción explícita o implícita (Echeburúa \& de Corral, 2006).

En Chile, durante los últimos años, ha existido un aumento gradual de las denuncias por delitos sexuales. Si se consideran solo las denuncias formales realizadas entre el 2006 y 2013 la cifra sobrepasa las 150.000 (Fundación Paz Ciudadana, 2014), siendo más de la mitad de las víctimas menores de edad (Subsecretaría de Prevención del Delito, 2015), lo que ilustra la focalización de este tipo de problemática en la población infanto-juvenil. Esta cifra sin embargo, podría extenderse ampliamente si se toma en cuenta la alta proporción de niños que no denuncian este tipo de situaciones traumáticas, llegando algunos autores a estimarla alrededor del 50\% (Echeburúa \& Guerricaechevarría, 2005).

Los estudios retrospectivos permiten tener una estimación de la frecuencia del ASI más allá de la cifra negra. Por ejemplo, un estudio realizado con adultos en Estados Unidos señala un $27 \%$ de las mujeres frente a un $16 \%$ de los hombres sufren abuso sexual de niños (Finkelhor, Hotaling, Lewis \& Smith, 1990). En tanto, en España la cifra de universitarios que reporta haber sufrido ASI alcanza al $8,4 \%$ en el caso de los hombres y al $13,2 \%$ en el de las mujeres (Cortés, Cantón \& Cantón-Cortés, 2011). En esta línea, diversos estudios chilenos buscan delimitar la frecuencia del ASI. Por ejemplo, Ysern y Becerra (2006), reportan que $9 \%$ de los estudiantes de 3o medio encuestados indica haber sufrido abuso sexual intrafamiliar durante su vida. Otro estudio chileno más reciente reporta que el $7 \%$ de los niños y niñas reconoce haber vivido algún tipo de violencia sexual en su vida. Haciendo la diferenciación por género, el mismo estudio reporta que el $12,8 \%$ de las niñas y el $3,3 \%$ de los niños han sufrido algún tipo de abuso sexual (Dirección de Estudios Sociales Universidad Católica [DESUC], 2008).

Más allá de la prevalencia del fenómeno, tanto a nivel internacional como en Chile, se han descrito las consecuencias que el ASI tiene para las personas que lo sufren. Las víctimas de ASI experimentan una serie de síntomas a corto plazo que están ampliamente documentados. Destacan así la depresión, la ansiedad, el estrés postraumático y el retraimiento social (Arredondo, 2002; Echeburúa \& Guerricaechevarría, 2005). En tanto, a largo plazo se ha descrito que las víctimas ASI reportan un menor ajuste psicológico que las personas que no han sido victimizadas, específicamente se observan mayores índices de depresión y una autoestima más deteriorada (Cantón \& Justicia, 2008). Más específicamente, un estudio chileno reportó la sintomatología de niños víctimas de ASI: el $85 \%$ presentaba síntomas ansiosos, el $57 \%$ sintomatología depresiva, el $49 \%$ de trastornos del sueño, el $49 \%$ dificultades de atención, el $43 \%$ conductas evitativas, el $36 \%$ comportamiento agresivo (tanto autoagresivo como heteroagresivo), el $28 \%$ tenía dificultades para el control de impulsos, el $26 \%$ tenía alteración de la conducta sexual normal, el $23 \%$ presentaba distorsiones cognitivas y el $23 \%$ presentaba trastornos a nivel de la conducta alimentaria (Navarro, Carrasco \& Aliste, 2003).

La visibilidad del ASI en Chile y la intervención reparatoria

Dada la alta prevalencia del fenómeno y la evidencia de las consecuencias negativas para los niños, en los últimos 20 años se ha avanzado en Chile en su consideración como un grave problema social. Permitiendo así el auge de centros especializados en la atención del ASI en todo el país.

Desde la ratificación de la Convención Internacional de los Derechos del Niño por parte del Estado de Chile en 1990, la política pública chilena favorece la visualización de las distintas formas de vulneración de la infancia y la adolescencia así como la generación de distintas estrategias de enfrentamiento de dichas vulneraciones. De esta forma, en 1990 el Gobierno de Chile elabora el primer Plan Nacional de Protección de la Infancia 1990-2000 seguido del correspondiente al período 2001-2010 (Ministerio de Planificación y Cooperación, 2010). Los objetivos centrales de estos planes fueron plantear e implementar diferentes líneas de acción dirigidas a resguardar los Derechos del Niño y del Adolescente, estableciendo como prioridad las diferentes situaciones de violencia contra los niños, especialmente en el contexto familiar. En este marco se articulan los cambios a la legislación nacional vigente que, en el caso de la violencia sexual infantil, implica importantes modificaciones a la ley relativa a los delitos sexuales (Ministerio de Justicia, 1999). Específicamente, se incorporan nuevos tipos penales, se redefinen las penas asociadas a ellos y se modifican algunos procedimientos investigativos (Servicio Nacional de Menores [SENAME], 2004).

Por su parte, en el ámbito de la intervención, surge en Chile en 1997 la primera línea programática especializada en intervención de tipo reparatoria de la violencia sexual infantil desde el Servicio Nacional de Menores (SENAME). Este hecho ilustra el interés del Estado por asumir la reparación del daño asociado a esta problemática $\mathrm{y}$, evidencia la necesidad de contar con dispositivos técnicos especializados para abordar esta tarea. Desde ese año hasta la actualidad, esta línea programática se ha ido adaptando en relación a las características del sujeto de atención, los objetivos de la intervención y sus principios metodológicos. Actualmente, este tipo de intervención se inscribe en la denominada Línea de Programas de Protección Especializada en Modalidad Maltrato Infantil Grave y Abuso Sexual (SENAME, 2012). Esta línea define el problema de atención desde el marco jurídico penal, identificando el ASI como delito. El objetivo general de esta línea de intervención se estipula como "contribuir al proceso reparatorio del niño, niñas o adolescente que ha sufrido maltrato físico grave y/o agresión 
sexual infantil" (SENAME, 2012, p. 2) e, integra como objetivos específicos a) la interrupción de la situación de agresión, b) la resignificación de la experiencia abusiva y c) el fortalecimiento de los recursos familiares y sociales. En este punto, cabe señalar que la conceptualización de la reparación, desde esta normativa, se refiere a la resignificación del daño asociado a la experiencia de maltrato grave o agresión sexual y se enfatiza tanto en la superación de las secuelas psicoafectivas como en la reelaboración de la experiencia abusiva (SENAME, 2012). Se espera que la intervención reparatoria, desde la categoría de superación de las secuelas psicoafectivas, se oriente a lograr que el niño asimile y se acomode a la experiencia vivida, utilizando esquemas cognitivos, conductuales y psicosociales. A través de este proceso se lograría la disminución o superación de los trastornos conductuales, de aprendizaje, sintomatología emocional, y superación de mecanismos de defensa inadecuados. Por su parte, en relación a la categoría de reelaboración, se espera que el niño o niña comprenda la dinámica del abuso, se reconozca como víctima y diferencie la responsabilidad comprometida en los hechos, eliminando las justificaciones del abuso. Para este proceso, se contempla un período de intervención multidisciplinaria en un plazo de entre 6 a 12 meses (SENAME, 2012).

Actualmente en Chile existen más de 70 programas especializados en la atención de víctimas de maltrato infantil grave y abuso sexual, con presencia en las 15 regiones del país (SENAME, 2014). Cada año se implementan nuevos centros especializados dado que la mayoría de los centros cuenta con lista de espera para la atención. A esta línea se suman otras propuestas técnicas en el ámbito público y privado, algunas de ellas son los Centros de Atención a Víctimas de Delitos Violentos de las Corporaciones de Asistencia Judicial del país. Se crean en 1994 con el objetivo de entregar apoyo psicológico, psiquiátrico, social y legal tanto a las víctimas directas de abuso sexual infantil como a sus familias (Corporación de Asistencia Judicial de Valparaíso, 2007). También se encuentra el Centro de Atención a Víctimas de Atentados Sexuales (CAVAS) dependiente de la Policía de Investigaciones de Chile, creado el año 1987, cuyo trabajo se enmarca en la labor de reparación del daño asociado al atentado sexual (CAVAS, 2003).

\section{Investigación referida a la psicoterapia con víctimas de ASI}

En base a estos antecedentes, es posible señalar que este conjunto de programas constituyen un enorme avance en el enfrentamiento del problema del ASI en Chile. No obstante, es necesario cuestionar sí este auge de centros ha ido a la par de la construcción de conocimiento en base a la sistematización de sus prácticas así como investigación que oriente la labor de los psicólogos clínicos de estos pacientes. El objetivo del presente estudio es describir la realidad chilena respecto a la elaboración y publicación de investigaciones vinculadas a la práctica de la psicoterapia con víctimas de ASI en medios de alta visibilidad. Es importante conocer la realidad chilena, dado que se han señalado diferencias culturales en torno al fenómeno del ASI que debieran tomarse en cuenta a la hora de planificar las intervenciones (Pereda, 2006).

A nivel internacional, existen diversos enfoques en el estudio de la práctica de la psicoterapia con víctimas de ASI. Varios estudios se centran en los factores que pudiesen nutrir el trabajo psicoterapéutico. Por ejemplo, dado que la sintomatología en distintas víctimas presenta una variación importante (Echeburúa \& Guerricaechevarría, 2005), una serie de estudios aportan modelos explicativos sobre las condiciones específicas más probables que conducen a un cierto tipo de sintomatología en los niños víctimas de ASI. Estos estudios hacen referencia a variables que median o moderan la relación entre la experiencia de ASI y la presencia de sintomatología. Uno de los modelos de mayor aceptación internacional es el de Finkelhor y Browne (1985), que destacan la sexualización traumática, la sensación de traición, la impotencia y la estigmatización del niño como las variables que median entre el ASI y sus consecuencias. Este tipo de estudios entrega importante información a la hora de diseñar las intervenciones reparatorias pues destaca elementos relevantes a considerar en ellas y, por tanto, es necesario conocer si existen estudios chilenos al respecto.

Por otro lado, la tradición investigativa de la psicoterapia basada en la evidencia empírica busca identificar tratamientos específicos apropiados para tratar trastornos psicológicos determinados. Esta línea propone procedimientos para evaluar y clasificar los tratamientos según la evidencia de su efectividad (Fernández \& Pérez, 2001). Por ejemplo, se tiene evidencia de que los tratamientos basados en la exposición (Baguena, 2001) y de que la terapia cognitivo conductual centrada en el trauma (Echeburúa \& Guerricaechevarría, 2011) son efectivos para enfrentar eventos traumáticos. En esta línea, tras realizar un meta-análisis que incluye la revisión de 33 artículos, Sánchez-Meca, RosaAlcázar y López-Soler (2011) señalan que los tratamientos cognitivo conductuales centrados en el trauma, combinados con terapia de contención y con elementos psicodinámicos (ej. terapia de juego) son los que obtienen mejores resultados en la disminución de sintomatología asociada al ASI (principalmente en las conductas sexualizadas, la ansiedad, la depresión, los problemas de autoestima y los problemas de conducta). Por ello, es necesario cuestionarse en el contexto nacional la existencia o no de investigación que respalde la efectividad de los procedimientos empleados por los psicoterapeutas chilenos.

Por último, se ha estudiado la incidencia de los factores comunes de los distintos modelos psicoterapéuticos y su incidencia en la efectividad de la psicoterapia. Desde esta perspectiva, más que la técnica específica utilizada por el terapeuta, tienen mayor incidencia en el cambio psicoterapéutico una 
serie de factores comunes a cualquier tipo de psicoterapia (alianza terapéutica, recursos de cliente y expectativas de éxito) (Lambert, 1991). En el contexto del ASI, distintos estudios indican que, pese al trauma experimentado, las víctimas no presentarían dificultades para formar una sólida alianza, (Callahan, Price \& Hilsenroth, 2003; Keller, Zoellner \& Feeny, 2010). Además, se ha estudiado la influencia positiva de la alianza en el logro terapéutico (Cloitre, Stovall-McClough, Miranda \& Chemtob, 2004). Estos estudios dan cuenta de que la alianza es un importante factor asociado al logro terapéutico (Paivio \& Patterson, 1999). No obstante, existe escaso conocimiento, incluso a nivel internacional, respecto de los factores que influyen la conformación de dicha alianza. Igualmente, sobre la incidencia de los otros factores comunes en la psicoterapia con víctimas de ASI. En consecuencia, resulta necesario profundizar en la materia (Callahan et al., 2003).

\section{Método}

\section{Muestra}

Se trabajó con una muestra intencionada de organismos que publican en la línea de la psicoterapia y del trabajo con víctimas de ASI. Los organismos seleccionados fueron ocho revistas de psicología y seis instituciones vinculadas a la atención o protección de víctimas de ASI. En particular, se trabajó con las siete revistas chilenas pertenecientes a la Asociación Chilena de Revistas Científicas de Psicología (Cuadernos de Neuropsicología, Psykhe, Summa Psicológica, Praxis, Revista de Psicología de la Universidad de Chile, Psicoperspectivas, Salud y Sociedad) y con la revista de la Sociedad Chilena de Psicología Clínica (Terapia Psicológica). Por su parte, las instituciones fueron seleccionadas por su antigüedad y cobertura en temáticas relacionadas con el ASI. Las instituciones consideradas fueron: Corporación Paicabi; Corporación de Asistencia Judicial (CAJ); Corporación Raíces; CAVAS; Corporación Opción; SENAME.

Dentro de estas fuentes, se seleccionaron los artículos chilenos referidos a la investigación en psicoterapia con víctimas de ASI. El criterio utilizado fue el siguiente: contenido referenciado como víctima de $\mathrm{ASI}$; relacionados con la psicoterapia o intervención psicológica; contexto nacional (autores chilenos y/o realizados con muestras chilenas); y disponibilidad en las páginas web de los organismos que los publican.

\section{Procedimiento}

En el caso de las revistas seleccionadas, en cada una de las páginas webs, el criterio de búsqueda se centró en "abuso sexual", "agresión sexual", "trauma" y "violación". Con dicho criterio, se revisó cada uno de los artículos resultantes. Solo se contabilizaron aquellos artículos vinculados a víctimas de abuso sexual infantil y no de otros sucesos (ej. violaciones a los Derechos Humanos, trauma por desastres naturales). En cuanto a las páginas web de las instituciones seleccionadas, la sección "publicaciones" disponible hasta abril de 2015 de cada una fue revisada en búsqueda de trabajos referidos a la práctica de psicoterapia con víctimas de ASI. Los artículos que vinculaban la psicoterapia con el abuso sexual fueron seleccionados y clasificados según la metodología empleada (sistematización de experiencias; análisis de caso; e investigación empírica) y según su foco (análisis de variables que inciden en la victimización y en la reparación; propuesta de modelos de intervención; análisis de los factores comunes y su incidencia en la psicoterapia).

\section{Resultados}

En primer lugar, se describen los artículos vinculados a la investigación en psicoterapia con víctimas de ASI de las revistas e instituciones elegidas. Seguidamente, se describe el tipo de estudio según su metodología y foco.

\section{Revisión de los artículos publicados en revistas científicas chilenas}

La revista Cuadernos de Neuropsicología ha publicado 17 números desde el año 2007. Solo 1 artículo vinculado al abuso sexual infantil (BernateNavarro, Baquero-Vargas \& Soto-Pérez, 2009) resulta de la búsqueda, pero no está referido a la psicoterapia ni está realizado en Chile. En consecuencia, no hay artículos publicados en esta revista vinculados a la investigación en psicoterapia con víctimas de ASI en Chile.

La revista Psykhe ha publicado 44 números desde el año 1992. De los 8 artículos vinculados al ASI resultantes, 5 de ellos no están referidos a la investigación en psicoterapia (Araya, 2003; Bustos, Rincón \& Aedo, 2009; Martínez, 2000; Pool, 2006, 2007) y 1 no está realizado con muestra chilena (Bacigalupe, 2009). Solamente 2 de los artículos publicados se refieren a la psicoterapia vinculada al ASI, pero no son de carácter empírico. Se trata del artículo de Llanos y Siclair (2001) que propone un modelo de trabajo psicoterapéutico para víctimas de abuso sexual y, del artículo de Sinclair y Martínez (2006) sobre la psicoterapia con las madres de niños abusados sexualmente y su efecto en las propias víctimas.

Por su parte, la revista Summa Psicológica ha publicado 21 números desde el año 2003. De los 6 artículos resultantes, encontramos 1 que consiste una revisión teórica (Pinto-Cortés, 2004), 1 en una caracterización de las víctimas de ASI (Gómez, Cifuentes \& Sieverson, 2010) y otro referido a un instrumento de medición del estrés postraumático (Guerra, Martínez, Ahumada \& Díaz, 2013). Los 3 artículos restantes si se vinculan de forma más directa con la psicoterapia: El artículo de Rodríguez, Basualto y Vivanco (2004), que analiza un caso clínico de una paciente víctima de abuso sexual incestuoso; el artículo de Calvo, Guerra, Martínez y Viveros (2010), que describe una intervención multidisciplinaria en un caso de violación transgeneracional; y por último, el trabajo de Plaza, Beraud y Valenzuela (2014), que estudia 40 víctimas 
de ASI y concluye que abusos sexuales intrafamiliares, crónicos y violentos son los que se asocian a sentimientos de estigmatización, indefensión, traición y culpa en las víctimas.

La revista Praxis cuenta con 25 números desde el año 1999. Solo 2 artículos están vinculados al abuso sexual infantil, no obstante, uno de ellos da cuenta de elementos periciales en ASI (Contreras \& Maffioletti, 2002). El único artículo más cercano a la psicoterapia es el de Cañas (2013) quién, luego de entrevistar a 8 madres de niños abusados sexualmente analiza los significados asociados a su incredulidad respecto a la existencia de ASI y su implicación en la psicoterapia reparatoria.

La revista de psicología de la Universidad de Chile ha publicado 40 números desde el año 1992. De los 7 artículos resultantes de la búsqueda, 2 no se relacionan directamente con el ASI si no que abordan el fenómeno más general del maltrato infantil (Aracena, Balladares, Román \& Weiss, 2002; Florenzano et al., 2005). 1 se relaciona con el ASI y los indicadores de los test gráficos, pero no con la psicoterapia (Opazo \& Rivera, 2010). Los 4 restantes si dan cuenta de investigaciones en el ámbito de la psicoterapia con víctimas de ASI: Guerra y Plaza (2009) ilustran el uso de un tratamiento cognitivo conductual para disminuir la sintomatología de estrés postraumático en un caso de violación infantil; Guerra, Viveros, Calvo, Canessa \& Mascayano (2011) evalúan los efectos de un programa de preparación a víctimas de ASI que deben declarar en juicios orales; Espinoza, Förster y Capella (2011) se aproximan al tratamiento de los hermanos de las víctimas de $\mathrm{ASI}$, entendiéndolos como víctimas indirectas; y Álvarez, Socorro y Capella (2012) evalúan el efecto de la psicoterapia de madres de víctimas de ASI en el proceso reparatorio de sus hijos.

Por su parte, desde el 2001 la revista Psicoperspectivas ha publicado 21 números. Encontrándose solamente el estudio de Castaldi (2014) con una propuesta de los elementos a considerar en la psicoterapia con víctimas de ASI.

En tanto, la revista Salud y Sociedad no registra artículos vinculados a la investigación en psicoterapia con víctimas de ASI en ninguno de los 15 números publicados desde el 2010.

La revista Terapia Psicológica cuenta con 27 números publicados disponibles en su página web desde el 2003 pese a publicar su primer artículo en la década de los 80 . De los 3 artículos resultantes, 1 no fue realizado en Chile (Sarasua, Zubizarreta, De Corral \& Echeburúa, 2012) mientras que 2 si cumplen con los criterios de selección: Ibaceta (2007) que relaciona los antecedentes de agresiones sexuales en la infancia con la psicopatología en la vida adulta y propone un marco general para el abordaje terapéutico; y Magaña, Ramírez y Menéndez (2014) quienes profundizan en las estrategias clínicas empleadas por 8 profesionales de atención a víctimas de ASI.

La tabla 1 ilustra las características de los 11 artículos chilenos publicados en las revistas elegidas que vinculan la investigación con la práctica de la psicoterapia con víctimas de ASI.

Tabla 1. Artículos que vinculan la investigación con la práctica de la psicoterapia con víctimas de ASI.

\begin{tabular}{|c|c|c|c|c|}
\hline Autores & Revista & Tipo de artículo & Metodología & $\begin{array}{l}\text { Tamaño } \\
\text { muestra }\end{array}$ \\
\hline $\begin{array}{l}\text { Llanos y Siclair } \\
(2001)\end{array}$ & Psykhe & Modelo de intervención & $\begin{array}{l}\text { Sistematización de } \\
\text { experiencias }\end{array}$ & 0 \\
\hline $\begin{array}{l}\text { Sinclair y } \\
\text { Martínez (2006) }\end{array}$ & Psykhe & Modelo de intervención & $\begin{array}{l}\text { Sistematización de } \\
\text { experiencias }\end{array}$ & 0 \\
\hline $\begin{array}{l}\text { Rodríguez et al. } \\
\text { (2004) }\end{array}$ & $\begin{array}{l}\text { Summa } \\
\text { Psicológica }\end{array}$ & Modelo de intervención & Análisis de caso & 1 \\
\hline $\begin{array}{l}\text { Calvo et al. } \\
(2010)\end{array}$ & $\begin{array}{l}\text { Summa } \\
\text { Psicológica }\end{array}$ & Modelo de intervención & Análisis de caso & 1 \\
\hline $\begin{array}{l}\text { Plaza et al. } \\
(2014)\end{array}$ & $\begin{array}{l}\text { Summa } \\
\text { Psicológica }\end{array}$ & $\begin{array}{l}\text { Variables que inciden } \\
\text { en terapia }\end{array}$ & Empírico & 40 \\
\hline Cañas (2013) & Praxis & $\begin{array}{l}\text { Variables que inciden } \\
\text { en terapia }\end{array}$ & Empírico & 8 \\
\hline $\begin{array}{l}\text { Álvarez et al. } \\
(2012)\end{array}$ & Revista U. Chile & Modelo de intervención & Empírico & 12 \\
\hline $\begin{array}{l}\text { Guerra y Plaza } \\
(2009)\end{array}$ & Revista U. Chile & Modelo de intervención & Análisis de caso & 1 \\
\hline $\begin{array}{l}\text { Guerra et al. } \\
\text { (2011) }\end{array}$ & Revista U. Chile & Modelo de intervención & Empírico & 8 \\
\hline
\end{tabular}




$\begin{array}{lllll}\begin{array}{l}\text { Espinoza et al. } \\ (2011)\end{array} & \text { Revista U. Chile } & \begin{array}{l}\text { Variables que inciden } \\ \text { en la terapia }\end{array} & \text { Empírico } & 8 \\ \text { Castaldi (2014) } & \begin{array}{l}\text { Psico_- } \\ \text { perspectivas }\end{array} & \text { Modelo de intervención } & \begin{array}{l}\text { Sistematización de } \\ \text { experiencias }\end{array} & 0\end{array}$

\section{Revisión de los artículos publicados por instituciones chilenas especializadas en trabajo con ASI}

Con respecto a las publicaciones realizadas por las instituciones seleccionadas, la Corporación de Promoción y Apoyo a la Infancia Paicabí cuenta con 6 libros sobre maltrato y abuso sexual infantil (Arredondo, 2002; Arredondo \& Toro 2007; Arredondo \& Toro, 2010; Arredondo, Knaak, Lira, Silva \& Zamora, 1998; Centro Antú, 2006; PUCV \& Paicabí, 2008). Solo 4 trabajos vinculan de alguna manera la investigación en psicoterapia con víctimas de ASI: El libro de Arredondo (2002) ofrece una guía para la prevención del ASI; el artículo de González y Guerra (2007) presenta un modelo de intervención psicológica con víctimas de ASI; el artículo de Canessa y Guerra (2010) que propone un programa de acompañamiento psicológico para víctimas que deben declarar en juicios orales; y el de Novoa, Benguria y Guerra (2010) que estudia la relación entre las expectativas terapéuticas de éxito en la terapia y el logro terapéutico en pacientes adolescentes víctimas de abusos sexuales.

Por su parte la Corporación de Asistencia Judicial tiene un libro publicado. En él, solo el artículo de Muñoz (2007) aborda la problemática estudiada, analizando algunas consideraciones para el trabajo con víctimas adolescentes.
La Corporación Raíces ha publicado más de 20 trabajos, principalmente centrados la reflexión en torno a la problemática de la explotación sexual comercial infantil. En general, estos trabajos versan sobre elementos reflexivos o sobre materiales psicoeducativos para comprender la problemática. El trabajo de Araya, Almendras, Ramírez, Vásquez y Magun (2006) es el más cercano a la psicoterapia propiamente, dando cuenta de experiencias de intervención reparatoria integral con las víctimas.

De forma similar, en las publicaciones de la Corporación Opción, destaca el trabajo que sistematiza un modelo de intervención reparatorio con víctimas de explotación sexual comercial infantil, desde la perspectiva de género (Corporación Opción, 2007).

CAVAS por su parte ha publicado un libro donde aparece el modelo de intervención sistematizado a partir de la experiencia en la atención de víctimas de ASI (CAVAS, 2003).

Por último, en la página web de SENAME figuran más de 50 trabajos, principalmente informes estadísticos, pero no se aprecian estudios referidos a la psicoterapia con víctimas de ASI.

Tabla 2. Trabajos de organizaciones que vinculan la investigación con la práctica de la psicoterapia con víctimas de ASI.

\begin{tabular}{|c|c|c|c|c|}
\hline Autores & Organismo & Tipo de artículo & Metodología & $\begin{array}{l}\text { Tamaño } \\
\text { muestra }\end{array}$ \\
\hline Arredondo (2002) & Paicabí & $\begin{array}{l}\text { Modelo de } \\
\text { intervención }\end{array}$ & $\begin{array}{l}\text { Sistematización de } \\
\text { experiencias }\end{array}$ & 0 \\
\hline $\begin{array}{l}\text { González y Guerra } \\
(2007)\end{array}$ & Paicabí & $\begin{array}{l}\text { Modelo de } \\
\text { intervención }\end{array}$ & $\begin{array}{l}\text { Sistematización de } \\
\text { experiencias }\end{array}$ & 0 \\
\hline $\begin{array}{l}\text { Canessa y Guerra } \\
(2010)\end{array}$ & Paicabí & $\begin{array}{l}\text { Modelo de } \\
\text { intervención }\end{array}$ & $\begin{array}{l}\text { Sistematización de } \\
\text { experiencias }\end{array}$ & 0 \\
\hline Novoa et al. (2010) & Paicabí & Factores comunes & Empírico & 64 \\
\hline Muñoz (2007) & CAJ & $\begin{array}{l}\text { Modelo de } \\
\text { intervención }\end{array}$ & $\begin{array}{l}\text { Sistematización de } \\
\text { experiencias }\end{array}$ & 0 \\
\hline Araya et al. (2006) & Raíces & $\begin{array}{l}\text { Modelo de } \\
\text { intervención }\end{array}$ & $\begin{array}{l}\text { Sistematización de } \\
\text { experiencias }\end{array}$ & 0 \\
\hline $\begin{array}{l}\text { Corporación Opción } \\
(2007)\end{array}$ & Opción & $\begin{array}{l}\text { Modelo de } \\
\text { intervención }\end{array}$ & $\begin{array}{l}\text { Sistematización de } \\
\text { experiencias }\end{array}$ & 0 \\
\hline CAVAS (2003) & CAVAS & $\begin{array}{l}\text { Modelo de } \\
\text { intervención }\end{array}$ & $\begin{array}{l}\text { Sistematización de } \\
\text { experiencias }\end{array}$ & 0 \\
\hline
\end{tabular}




\section{Análisis del foco y metodología de los trabajos publicados en revistas $y$ organismos especializados.}

Considerando las 19 publicaciones seleccionadas, se aprecia que la mayoría $(78,9 \%)$ corresponde a la propuesta de modelos de intervención mientras una menor proporción aborda el estudio de variables que pueden incidir en la psicoterapia $(15,8 \%)$ o en el análisis de la importancia de los factores comunes en la psicoterapia con víctimas de ASI (5,3\%).

Respecto a la metodología empleada, mayormente se corresponde a la sistematización de experiencias o prácticas de intervención (52,6\%) y en una menor proporción se encuentran los estudios empíricos $(31,6 \%)$ y los análisis de caso único $(15,8 \%)$. Destaca el reducido tamaño de la muestra (entre 8 y 64 personas) de los pocos estudios empíricos realizados en el área publicados en las fuentes seleccionadas.

\section{Discusión}

El objetivo del presente estudio se centra en describir la realidad chilena respecto a la elaboración y publicación de investigaciones vinculadas a la práctica de la psicoterapia con víctimas de ASI en una muestra de organismos que publican este tipo de trabajos. De los resultados, se observa el número escaso de publicaciones disponibles en los medios seleccionados (19 publicaciones en 14 revistas $u$ organizaciones de amplia trayectoria en el ámbito de la psicología y atención de víctimas). Sin embargo, una de las principales limitaciones de este estudio reside en la no revisión de otras fuentes de investigación como sería el caso de tesis de grado.

Si bien cada uno de los trabajos descritos aporta información y reflexión importante para el conocimiento del ASI, la investigación empírica referida a la psicoterapia con este tipo de víctimas es escasa. Mayoritariamente se aborda la sistematización de experiencias de intervención generales y las muestras son reducidas resaltando así, la posible dificultad en acceder a muestras de víctimas infantiles, tanto por razones prácticas como éticas. Con lo cual se potencia la limitación de generalización de los resultados, aspecto de alta relevancia si se considera que la investigación empírica debiera nutrir el trabajo de los psicoterapeutas chilenos en este ámbito específico.

Respecto al foco de las publicaciones analizadas, se aprecia escasez de trabajos que aporten elementos para comprender las variables que inciden en el proceso de desarrollo de sintomatología y que son de alta relevancia para la psicoterapia (factores mediadores y moderadores de la sintomatología), así como aquellas que aporten elementos para comprender la incidencia de los factores comunes (alianza terapéutica, expectativas, recursos del paciente) en el éxito de la terapia en la línea planteada por Lambert (1991).

Esta insuficiencia de estudios muestra el desequilibrio entre la práctica de la intervención reparatoria (muy desarrollada a nivel nacional) y la investigación que debiera respaldarla. Si bien, las instituciones especializadas han hecho esfuerzos por sistematizar y publicar sus prácticas de intervención, las investigaciones empíricas que reporten datos fiables sobre la efectividad de estas intervenciones o de los factores que inciden en ello son escasas. Con el fin de generar mayor cantidad de estudios que abarquen mayor cantidad de focos y otorguen mayores garantías metodológicas en el área, se considera importante generar alianzas entre el mundo académico y las instituciones que atienden a las víctimas.

La insuficiencia de estudios en Chile acentúa la dificultad para los terapeutas de generar parámetros en el desarrollo de psicoterapias reparatorias. Por ello, es oportuno cuestionarse los criterios que fundamentan sus intervenciones. Los terapeutas posiblemente se basen en estudios realizados en otros contextos culturales o bien, en la propia experiencia clínica. Esta última, una estrategia valorable en el caso de los terapeutas con experiencia, pero extremadamente arriesgada en los novatos (Vélez \& Restrepo, 2008).

He aquí la necesidad de realizar esfuerzos por desarrollar investigaciones en el contexto nacional que aporten conocimiento relevante para la conformación de tratamientos eficientes y eficaces que den respuesta a la demanda de atención de las personas expuestas a este tipo de evento traumático en Chile.

Se estima que el aumento de las investigaciones impactará positivamente en los usuarios, las víctimas de ASI y sus familias, al participar de procesos reparatorios diseñados y validados en un contexto cultural similar a los mismos. No se trata de construir "recetas para la intervención" ya que es sabido que la complejidad del abuso es tan amplia que hace que cualquier intento por automatizar las intervenciones sea un inconveniente, sino de generar protocolos que guíen la intervención. Protocolos que estén respaldados empíricamente y que permitan al psicólogo clínico tomar decisiones que orienten su intervención con distintos tipos de pacientes.

El aumento de investigación en psicoterapias por ASI también impactará positivamente en la dificultad que experimentan los terapeutas para abordar el tratamiento del ASI dado su grado de complejidad (Barrera \& Guerra, 2007) y que a su vez se señala como un elemento asociado a su desgaste laboral.

Finalmente, resaltar la contribución también en el ámbito jurídico. Un aumento de investigaciones centradas en el ASI como materia de delito, tendrán un impacto positivo principalmente, dotando de herramientas básicas para comprender el fenómeno, la variedad de síntomas asociados y la disparidad en la evolución que presentan diferentes víctimas a legisladores, jueces, abogados y policías. Los mismos beneficios podrían aplicarse al ámbito preventivo, tanto en instituciones de diversa índole como en la comunidad en general. 


\section{Referencias}

Álvarez, P., Socorro, A. \& Capella, C. (2012). Influencia de una intervención grupal para madres en el cambio psicoterapéutico de sus hijos/as víctimas de agresiones sexuales. Revista de Psicología de la Universidad de Chile 21(2), 31-54. doi:10.5354/0719-0581.2012.25836

Aracena, M., Balladares, E., Román, F. \& Weiss, C. (2002). Conceptualización de las pautas de crianza de buen trato y maltrato infantil, en familias del estrato socioeconómico bajo: Una mirada Cualitativa. Revista de Psicología de la Universidad de Chile, 11(2), 39-53. doi:10.5354/0719-0581.2002.17286

Araya, C. (2003). Escala para Medir Creencias que Perpetúan la Violencia Intrafamiliar: Estudios Preliminares. Psykhe 12(1), 83-96. Recuperado de

http://www.psykhe.cl/index.php/psykhe/article/vi $\mathrm{ew} / 331 / 311$

Araya, D., Almendras, I., Ramírez, A., Vásquez, D. \& Magun, A. (2006). "Ya no tengo nada que esconder": experiencias de reparación con niños, Niñas y adolescentes víctimas de explotación sexual comercial. Santiago: Andros.

Arredondo, V. (2002). Guía básica de prevención del abuso sexual infantil. Valparaíso: Corporación Paicabí.

Arredondo, V. \& Toro, E. (2007). Violencia sexual infantil: debates, reflexiones y prácticas críticas. Valparaíso: Corporación Paicabí

Arredondo, V. \& Toro, E. (2010) Espejos de Infancia: Análisis e Intervenciones en violencia infantil. Valparaíso: Corporación Paicabí.

Arredondo, V., Knaak, M., Lira, G., Silva, A. \& Zamora, I. (1998). Maltrato Infantil: Elementos básicos para su comprensión. Viña del Mar: Corporación Paicabí.

Bacigalupe, G. (2009). Latinos Sobrevivientes de Abuso Sexual Infantil en los Estados Unidos: Un Acercamiento Relacional al Diagnóstico e Intervención. Psykhe 10(2), 167-180. Recuperado de http://www.psykhe.cl/index.php/psykhe/article/vi $\mathrm{ew} / 366$

Baguena, M. (2001). Tratamientos psicológicos eficaces para el estrés postraumático. Psicothema, 13(3), 479-492. Recuperado http://www.psicothema.es/pdf/470.pdf

Barrera, P. \& Guerra, C. (2007). Creencias de profesionales que atienden a Víctimas de Abuso Sexual Infantil respecto de la influencia del Proceso Legal en su Desgaste y Satisfacción Laboral. En Corporación de Asistencia Judicial (Ed.), Atención a Víctimas de Delitos Violentos: Reflexiones desde la Práctica (2001-2007) (pp. 177-192). Viña del Mar: RIL Editores.

Bernate-Navarro, M., Baquero-Vargas, M. \& Soto-Pérez, F. (2009). Diferencias en los Procesos de Atención y Memoria en Niños con y sin Estrés Postraumático. Cuadernos de Neuropsicología 3(1), 104-115. Recuperado de http://www.cnps.cl/index.php/cnps/article/view/7
Bustos, P., Rincón, P. \& Aedo, J. (2009). Validación preliminar de la Escala infantil de síntomas de estrés postraumático (Child PTSD Symptoms Scale, CPSS) en niños/as y adolescentes víctimas de violencia sexual. Psykhe, 18(2), 118126. doi:10.4067/S0718-222820090002000233

Canessa, P. \& Guerra, C. (2010). Programa de entrenamiento para niños victimizados sexualmente que deben declarar en un juicio oral: aportes de la terapia cognitivo conductual. En V. Arredondo \& E. Toro (Comps.), Espejos de Infancia: Análisis e Intervenciones en violencia infantil (pp. 89-106). Valparaíso: Corporación Paicabí.

Cantón, D. \& Justicia, F. (2008). Afrontamiento del abuso sexual infantil y ajuste psicológico a largo plazo. Psicothema, 20(4), 509-515. Recuperado de http://www.psicothema.com/resumen.asp?id=35 $\underline{15}$

Cañas, R. (2013). Madres incrédulas frente a la agresión sexual de su pareja hacia un hijo: Significados construidos en torno a la experiencia de incredulidad. Praxis, 24, 57-77. Recuperado de http://www. praxis.udp.cl/pdf/24/Praxis24-04.pdf

Callahan, K., Price, J. \& Hilsenroth, M. (2003). Psychological Assessment of Adult Survivors of Childhood Sexual Abuse within a Naturalistic Clinical Sample. Journal of Personalitty Assessment, $\quad$ 80(2), 173-184. doi:10.1207/S15327752JPA8002_06

Calvo, B., Guerra, C., Martínez, P. \& Viveros, M (2010). Atención Psicosociojurídica Integral en un Caso de Violación Transgeneracional. Summa Psicológica UST, 7(2), 25-34. Recuperado de http://summapsicologica.cl/index.php/summa/art icle/view/119

Castaldi, L. (2014). Psicoterapia con niños/as y adolescentes que han sido víctimas de agresiones sexuales: Sobre la reparación, la resignificación y la superación. Psicoperspectivas, 13(3), 93-105. Recuperado de

http://www.psicoperspectivas.cl/index.php/psico perspectivas/article/view/348

Centro Antú (2006). Los Secretos del Eclipse. Explotación Sexual Comercial Infantil. Sistematización de la experiencia del Centro Antú de la Corporación Paicabí 2004-2005. Valparaíso: Corporación Paicabí.

Centro de Asistencia a Víctimas de Atentados Sexuales (2003). Centro de Asistencia a Víctimas de Atentados Sexuales CAVAS Metropolitano: 16 años de Experiencia. Santiago, Chile: Policía de Investigaciones de Chile.

Cloitre, M., Stovall-McClough, C., Miranda, R. \& Chemtob, C. (2004). Therapeutic alliance, negative mood regulation, and treatment outcome in child abuse-related posttraumatic stress disorder. Journal of Consulting and Clinical Psychology, 72(3), 411-416. doi:10.1037/0022006X.72.3.411

Contreras, L. \& Maffioletti, F. (2002). Valoración psicojurídica de la veracidad del testimonio en la evaluación del abuso sexual infantil. Praxis, 4, 128-137.

Recuperado 
https://lasdisidentes.com/2013/04/01/valoracionpsico-juridica-de-la-veracidad-del-testimonio-enla-evaluacion-del-abuso-sexual-infantil//

Corporación de Asistencia Judicial. (2007). Atención a Víctimas de Delitos Violentos: Reflexiones desde la Práctica. Viña del Mar: RIL Editores.

Corporación Opción. (2007). Caracterización y Sistematización de las Experiencias de Niños, Niñas y Adolescentes Víctimas de ESCI. Modelo de Intervención Reparatorio desde una Perspectiva de Género. Recuperado de http://opcion.cl/wpcontent/uploads/2007/03/Investigacion ESCl la uique.pdf

Cortés, R., Cantón; J. \& Cantón-Cortés, D. (2011). Naturaleza de los abusos sexuales a menores y consecuencias en la salud mental de las víctimas. Gaceta Sanitaria, 25(2), 157-165. doi:10.1016/j.gaceta.2010.10.009

Dirección de Estudios Sociales Universidad Católica. (2008). Encuesta Nacional de Victimización por Violencia Intrafamiliar y Delitos Sexuales. Ministerio del Interior. Recuperado de http://estudios.sernam.cl/?m=e\&l=151

Echeburúa, E. \& de Corral, P. (2006). Secuelas emocionales en víctimas de abuso sexual en la infancia. Cuaderno Medicina Forense, 12(4344), 75-82. Recuperado de http://scielo.isciii.es/pdf/cmf/n43-44/06.pdf

Echeburúa, E. \& Guerricaechevarría, C. (2005). Concepto, factores de riesgo y efectos psicopatológicos del abuso sexual infantil. En J. Sanmartín (Ed.), Violencia contra los niños (pp. 86-112). Barcelona: Ariel.

Echeburúa, E. \& Guerricaechevarría, C. (2011). Tratamiento psicológico de las víctimas de abuso sexual infantil intrafamiliar: un enfoque integrador. Psicología Conductual, 19(2), 469486. Recuperado http://www.ehu.eus/echeburua/pdfs/ASI.pdf

Espinoza, Y., Förster, C. \& Capella, C. (2011). Hermanos de víctimas de agresiones sexuales: una aproximación a las víctimas indirectas. Revista de Psicología de la Universidad de Chile 20(2), 77-102. doi:10.5354/0719-0581.2011.17933

Fernández, J. \& Pérez, M. (2001). Separando el grano de la paja en los tratamientos psicológicos. Psicothema, 13(3), 337-344. Recuperado de http://www.psicothema.com/psicothema.asp?id= $\underline{458}$

Finkelhor, D. \& Browne, A. (1985). The traumatic impact of child abuse: A conceptualization. American Journal of Orthopsychiatry, 55(4), 530-541. doi:10.1111/j.1939-0025.1985.tb02703.x

Finkelhor, D., Hotaling, G., Lewis, I. \& Smith, C. (1990). Sexual abuse in a national survey of adult men and women: Prevalence, characteristics and risk factors. Child Abuse \& Neglect, 14(1), 19-28. doi:10.1016/0145-2134(90)90077-7

Florenzano, R., González, I., Errázuriz, P., Ventura, R., Gibbons, A., Blumel, B., ... \& Carvajal, C. (2005). Percepción de Funcionalidad Familiar y Trauma Infanto- juvenil: un estudio cualitativo. Revista de
Psicología de la Universidad de Chile, 14(2), 7992. doi:10.5354/0719-0581.2005.17424

Fundación Paz Ciudadana. (2014). Balance de la Delincuencia 2013. Recuperado de http://www.pazciudadana.cl/wpcontent/uploads/2015/01/balance-2013.pdf

Gómez, E., Cifuentes, B. \& Sieverson, C. (2010). Características asociadas al abuso sexual infantil en un programa de intervención especializada en Santiago de Chile. Summa Psicológica UST, 7(1), 91-104. Recuperado de http://summapsicologica.cl/index.php/summa/art icle/view/114

González, D. \& Guerra, C. (2007). Atención a víctimas de explotación sexual comercial infantil: El modelo de intervención psicológica del Centro Antú. En V. Arredondo \& E. Toro (Comp.), Violencia sexual infantil: debates, reflexiones y prácticas críticas (pp. 220-230). Valparaíso: Corporación Paicabí.

Guerra, C. \& Plaza, H. (2009). Tratamiento cognitivo conductual del estrés postraumático en un caso de violación infantil. Revista de Psicología de la Universidad de Chile, 18(1), 103-129. doi:10.5354/0719-0581.2009.17130

Guerra, C., Martínez, P., Ahumada, C. \& Díaz, M. (2013). Análisis psicométrico preliminar de la Escala de trauma de Davidson (DTS) en adolescentes chilenos. Summa Psicológica UST, 10(2), 41-48. Recuperado http://summapsicologica.cl/index.php/summa/art icle/view/139

Guerra, C., Viveros, M., Calvo, B., Canessa, P. \& Mascayano, F. (2011). Niveles de ansiedad en niños victimizados sexualmente que deben declarar en juicios orales: aportes de un programa de preparación. Revista de Psicología de la Universidad de Chile, 20(2), 7-24. doi:10.5354/0719-0581.2011.17930

Ibaceta, F. (2007). Agresión sexual en la infancia y viaje al futuro: Clínica y psicoterapia en la edad adulta. Terapia Psicológica, 25(2), 189-198. doi:10.4067/S0718-48082007000200010

Keller, S., Zoellner, L. \& Feeny, N. (2010). Understanding Factors Associated With Early Therapeutic Alliance in PTSD Treatment: Adherence, Childhood Sexual Abuse History, and Social Support. Journal of Consulting and Clinical Psychology, 78(6), 974-979. doi:10.1037/a0020758

Lambert, M. (1991). Introduction to psychotherapy research. En L. Beutler \& M. Crago (Eds.), Psychotherapy research: An international review of programmatic studies (pp. 1- 11). Washington, DC: American Psychological Association.

Llanos, M. \& Sinclair, C. (2001). Terapia de reparación en víctimas de abuso sexual. Aspectos fundamentales. Psykhe, 10(2), 53-70. Recuperado de http://www.psykhe.cl/index.php/psykhe/article/vi $\mathrm{ew} / 373$

Magaña, I. Ramírez, C. \& Menéndez, L. (2014). Abuso Sexual Infantil (ASI): Comprensiones y Representaciones Clínicas desde las prácticas 
de Salud Mental. Terapia Psicológica, 32(2), 133-142. 48082014000200006 doi:10.4067/S0718-

Martínez, J. (2000). Prevención del Abuso Sexual Infantil: Análisis Crítico de los Programas Educativos. Psykhe, 9(2), 63-74. Recuperado de http://www.psykhe.cl/index.php/psykhe/article/vi $\underline{\mathrm{ew} / 443}$

Ministerio de Justicia. (1999). Modificaciones a la Ley $19617 . \quad$ Recuperado de http://www.leychile.cl/Navegar?idNorma=13881

Ministerio de Planificación y Cooperación. (2010). Política Nacional y Plan de Acción Integrado a Favor de la Infancia y Adolescencia. Gobierno de Chile. 2001-2010. Recuperado de http://www.oei.es/quipu/chile/politica_infancia.pd

Muñoz, N. (2007). Trauma sexual y adolescencia. Una aproximación al impacto de la experiencia de abuso sexual en el desarrollo adolescente. En Corporación de Asistencia Judicial (Ed.), Atención a Víctimas de Delitos Violentos: Reflexiones desde la Práctica (pp. 157-166). Viña del Mar: RIL Editores.

Navarro, C., Carrasco, E. \& Aliste, M. (2003). Diagnóstico psicosocial de menores víctimas de agresiones sexuales: Un estudio descriptivo. En Anales $V$ Congreso Iberoamericano de Psicología Jurídica (pp. 89-108). Santiago, Chile: Asociación lberoamericana de Psicología Jurídica \& Policía de Investigaciones de Chile.

Novoa, P., Benguria, A. \& Guerra, C. (2010). Relación entre las expectativas terapéuticas y el logro terapéutico: Un estudio en jóvenes víctimas de abuso sexual. En V. Arredondo \& E. Toro (Comps.), Espejos de Infancia: Análisis e Intervenciones en violencia infantil (pp. 152-172). Valparaíso: Corporación Paicabí.

Opazo, V. \& Rivera, J. (2010). Indicadores gráficos de la prueba del Dibujo de la Figura Humana en adolescentes hombres y mujeres de 12 a 16 años víctimas de agresiones sexuales. Revista de psicología de la Universidad de Chile, 19(1), 80-107. doi:10.5354/0719-0581.2010.17099

Paivio, S. \& Patterson, L. (1999). Alliance development in therapy resolving child abuse issues. Psicotherapy, 36(4), 342-354. doi:10.1037/h0087843

Pereda, N. (2006). Malestar psicológico en estudiantes universitarios víctimas de abuso sexual infantil y otros estresores (Tesis doctoral inédita). Universidad de Barcelona, España.

Pinto-Cortés, C. (2014). Resiliencia psicológica: Una aproximación hacia su conceptualización, enfoques teóricos y relación con el abuso sexual infantil. Summa Psicológica UST, 11(2), 19-33. doi:10.18774/summa-vol11.num2-129

Plaza, H., Beraud, C. \& Valenzuela, C. (2014). Procesamiento traumatogénico del abuso sexual infantil en niñas y su relación con variables victimológicas. Summa Psicológica, 11(2), 3544. doi:10.18774/summa-vol11.num2-184
Pontificia Universidad Católica de Valparaíso \& Paicabí. (2008). Investigación sobre abuso sexual infantil en la Región de Valparaíso. Valparaíso: SENAME.

Pool, A. (2006). Análisis Desde el Modelo Traumatogénico de los Indicadores Gráficos Asociados a Agresiones Sexuales Infantiles en la Prueba Persona Bajo la Lluvia. Psykhe, 15(1), 45-55. doi:10.4067/S0718-22282006000100004

Pool, A. (2006). Diagnóstico Estructural a Través de la Prueba Persona Bajo la Lluvia en Niños y Niñas de 9 a 11 Años de Edad, Víctimas de Agresiones Sexuales Crónicas. Psykhe, 16(2), 117-128. doi:10.4067/S0718-22282007000200010

Rodríguez, A., Basualto, C. \& Vivanco, C. (2004). Rol de las Parejas en el Proceso Terapéutico de Mujeres Sobrevivientes de Abuso Sexual Incestuoso. Summa Psicológica UST, 2(1), 4155. Recuperado de http://summapsicologica.cl/index.php/summa/art icle/view/20

Sánchez-Meca, J., Rosa-Alcázar, A. \& López-Soler, C. (2011). The psychological treatment of sexual abuse in children and adolescents: A metaanalysis. International Journal of Clinical and Health Psychology, 11(1), 67-93. Recuperado de http://www.aepc.es/ijchp/articulos pdf/ijchp371.pdf

Sarasua, B., Zubizarreta, I., De Corral, P. \& Echeburúa, E. (2012). Factores de Vulnerabilidad y de Protección del Impacto Emocional en Mujeres Adultas Víctimas de Agresiones Sexuales. Terapia Psicológica, 30(3), 7-18. doi:10.4067/S0718-48082012000300002

Servicio Nacional de Menores. (2004). Explotación Sexual Comercial Infantil. Recuperado de http://www.sename.cl/wsename/otros/doc sena me/Explo sexual com inf.pdf

Servicio Nacional de Menores. (2012). Bases Técnicas Específicas Programas de Protección Especializada. Modalidad Maltrato Infantil Grave y Abuso Sexual. Recuperado de http://www.sename.cl/wsename/otros/proteccion BTTE.pdf

Servicio Nacional Menores. (2014). Catastro de la Oferta Programática de la Red Sename. Recuperado de http://www.sename.cl/wsename/otros/op/CATA STRO-201401.pdf

Sinclair, C. \& Martínez, J. (2006). Culpa o Responsabilidad: Terapia con Madres de Niñas y Niños que han Sufrido Abuso Sexual. Psykhe, 15(2), 25-35. doi:10.4067/S0718-222820060002000309

Subsecretaría de Prevención del Delito. (2015). Víctimas Constatadas en los partes de Denuncias y Detenciones. Recuperado de http://www.seguridadpublica.gov.cl/estadisticas/ datos-por-sexo-y-edad/

Vélez, P. \& Restrepo, O. (2008). El proceso de terminación en psicoterapia de tiempo limitado: Aspectos clínicos y técnicos. Revista CES Psicología, 1(2), 58-68. Recuperado de http://revistas.ces.edu.co/index.php/psicologia/a rticle/view/80 
Ysern, J. \& Becerra, P. (2006). Abuso sexual: Prevalencia y Características en jóvenes de $3^{\circ}$ medio de liceos municipalizados de Chillán, Chile. Theoria,
15(1),
79-85.
Recuperado

http://www.ubiobio.cl/theoria/v/v15-1/a7.pdf

\section{de}

